May 1990

biotic efficacy has been shown for therapy with the intravenous anti-leukocyte integrin antibody in the animal model.

The therapies developed in experimental models have proved highly efficacious in humans, forming a strong experimental and clinical data base for considering recommendation of a new standard for therapy of bacterial meningitis in children and adults that includes anti-inflammatory agents. It remains to be determined which single agent or combination of agents will yield the most benefit. New trials in neonates will determine if this group will also derive benefit from adjunct therapy as predicted by experimental evidence. What is certain is that a new avenue finally exists to decrease the up to $30 \%$ mortality in children and $50 \%$ mortality in neonates who contract bacterial meningitis.

\section{Bibliography}

Giebink G, Batalden P, Le C, et al: A controlled trial comparing three treatments for otitis media with effusion. Pediatr Infect Dis J 9:33-40, 1990.

Grigis N, Farid Z, Mikhail I, et al: Dexamethasone treatment for bacterial meningitis in children and adults. Pediatr Infec Dis J 8:848-851, 1989.

Jacobs R: Gram-negative enteric meningitis in the neonate. Infect Dis Newsletter 8:60-62, 1989.

Lebel M, Freij B, Syroglannopoulos G: Dexamethasone therapy for bacterial meningitis: Results of two double-blind, placebo-controlled trials. N Engl J Med 3:964-971, 1988.
Meningitis Study Group: Report of a workshop: Pathophysiology of bacterial meningitis - Implications for new management strategies. Pediatr Infect Dis J 6:1143-1171, 1987.

Meningitis Study Group: Report of a second workshop: Pathophysiology of bacterial meningitis. Pediatr Infect Dis J 8:899-933, 1989.

Tuomanen E, Hengstler B, Rich R, et al: Nonsteroidal antiinflammatory agents in the therapy for experimental pneumococcal meningitis. J Infec Dis 155:985$990,1987$.

Tuomanen E, Saukkonen K, Sande S, et al: Reduction of inflammation, tissue damage, and mortality in bacterial meningitis in rabbits treated with monoclonal antibodies against adhesion-promoting receptors of leukocytes. J Exp Med 170:959-968, 1989.

\title{
Haemophilus influenzae Type B Conjugate Vaccines
}

\section{Janet R. Gilsdorf, MD}

Department of Pediatric Infectious Diseases, University of Michigan, C.S. Mott Children's Hospital, Ann Arbor, MI

Haemophilus influenzae type B is a major pathogen in children, causing serious infections such as meningitis, facial cellulitis, pneumonia, septic arthritis, and epiglottitis in approximately 20,000 children in the United States (U.S.) annually. In spite of the availability of good antibiotic therapy, the mortality rate of $H$. influenzae type B meningitis, the most lifethreatening of these infections, is $5 \%$ to $10 \%$. In addition, significant neurologic sequelae occur in many children following $H$. influenzae meningitis.

In order to prevent infection with $H$. influenzae type $\mathrm{B}$, several vaccines have been licensed for use in children. The first series of these vaccines, which were introduced in 1985, contained only the purified capsular material, polyribosylribose phosphate or PRP. Extensive experience with the plain PRP vaccines demonstrated their poor immunogenicity in young children $<24$ months of age. However, the majority of $H$. influenzae infections occur in younger children with the peak incidence at about 9 to 11 months of age. In attempting to develop vaccines that would be effective at younger ages, the newer $H$. influenzae type B vaccines have used the plain PRP polysaccharide conjugated to various proteins. The first $H$. influenzae type B protein conjugate vaccine became available in 1987 with the licensure PRP-D, which contained plain PRP conjugated to diphtheria toxoid, and has been marketed under the name PROHIBIT by Connaught Laboratories. More recently, PRP-CRM, which contains smaller PRP molecules or oligosaccharides, conjugated to a mutant diphtheria protein called CRM197 has been li- censed and is marketed as HIBTITER by Praxis Biologics. A third conjugate vaccine, PRP-NOMP, called PEDVAX-HIB and made by Merck, Sharp, and Dohme, has most recently been licensed by the Food and Drug Administration. This vaccine contains plain PRP conjugated to an outer membrane protein of Neisseria meningitidis group B. All three of the conjugate vaccines have been licensed by the FDA for use in 18- to 71-month-old children, with permissive use at 15 months.

A major concern in the development of new vaccine products is their safety. Experience with PRP-D has shown a few relatively minor reactions. The number and types of adverse reactions appear to be no different from those observed with the older, plain PRP vaccine, and include mild irritability and tenderness at the injection site in $<15 \%$ of recipients; and low-grade fever, mild erythema, and swelling at the injection site in $<2 \%$ of recipients. Less information is available on reactions associated with PRP-CRM and PRP-NOMP, but they appear to be no different from those following PRP-D. Thus, $H$. in- 
fluenzae type B conjugate vaccines appear to be very safe in terms of vaccine-related reactions.

Following licensure of the plain PRP vaccine, preliminary observations of vaccine failures suggested a possible increase in the frequency of $H$. influenzae type $\mathrm{B}$ infections during the first week after vaccination. These rare, early cases may have represented real vaccine-associated infections or merely the background incidence of $H$. influenzae infections. A few may also have resulted from high-risk situations, such as exposure to a child with $H$. influenzae infection. Although it is not clear that these infections were truly related to receipt of vaccine, a mechanism has been proposed to explain possible susceptibility to $H$. influenzae type $\mathrm{B}$ infection soon after vaccination. In individuals with preexisting antibody against PRP, a decrease in the level of PRP antibodies has been shown during the first several days following vaccination with plain PRP. This decrease is then followed by an increase in antibody as the vaccinee develops an immune response to the vaccine. The decline in PRP antibody level is probably due to the formation of immune complexes of preformed antibody and circulating vaccine antigen. Furthermore, the decline in serum antibodies has been correlated with the appearance of vaccine antigen in the urine, probably reflecting the presence of circulating vaccine antigen. PRP antigenuria has been observed immediately following vaccination with both PRP-D and PRP-NOMP. However, it has not been observed following PRP-CRM, possibly because the small carbohydrate in this vaccine is not recognized by the latex agglutination assay used to detect antigenuria.

In considering antibody levels following vaccination with the conjugate vaccines, some individuals who have preexisting $H$. influenzae antibodies have demonstrated a decrease in these antibodies following PRP-D and PRP-NOMP vaccination. Data have not been presented for review on PRP antibody levels following PRP-CRM vaccination. In terms of actual $H$. influenzae type $\mathrm{B}$ infections during the first week following conjugate vaccine use, only a very few early cases have occurred following PRP-D vaccine and they may represent background. Out of 52,000 children in the U.S. receiving PRP-D, 2 experienced $H$. influenzae type $\mathrm{B}$ disease on the second day following vaccination. In Finland, no cases were seen in the first week following PRP-D vaccination in 57,000 children. Neither PRP-CRM nor PRP-NOMP has been used extensively enough for an accurate assessment.

Another area of major concern in the evaluation of vaccines is efficacy. Two methods have been used to evaluate the efficacy of a vaccine: immunogenicity and protection. In studies of immunogenicity, pre- and postvaccine antibody levels are measured and compared. In protection studies, the vaccine is given and subsequent occurrence of disease is monitored. In evaluating $H$. influenzae type $\mathrm{B}$ vaccine, protection studies are logistically difficult because the disease is relatively infrequent and large numbers of children and long follow-up periods are required to completely evaluate protection. The ideal protection studies are the placebo-controlled trials such as those that have been done in Finland and in Alaska. Because of these logistic problems, the FDA has licensed the $H$. influenzae vaccines in the U.S. based on their immunogenicity; then postmarketing protection surveys have been conducted. Many questions remain concerning the correlation of immunogenicity and antibody levels with protection. A level of $>0.15$ $\mu \mathrm{g} / \mathrm{mL}$ of natural antibodies or $>1$ $\mu \mathrm{g} / \mathrm{mL}$ vaccine-induced antibodies has been suggested as reflecting protective levels. However, this is probably too simplistic. Usual antibody assays measure total anti-PRP antibody, but total antibody contains antibodies that differ in immunoglobulin class, in immunoglobulin $\mathrm{G}$ subclass, in binding affinity, and in epitope specificity. These parameters can vary considerably from child to child and recent Finnish data suggest a poor correlation between total antibody level and protection. Postmarketing evaluation of PRP-D and PRP-CRM is currently in progress. Vaccine failures appear to be much less common with PRP-D than with plain PRP; information is not yet available on the PRP-CRM.

A confusing observation with plain PRP vaccine was the variability of efficacy seen in several areas within the U.S. and also the lower efficacy in the U.S. than was expected based on the old Finland trials. In fact, PRP vaccine appeared to have no efficacy in Minnesota. More recent data show that, since the introduction of PRP-D, no vaccine failures have occurred in Minnesota. In summary, all $H$. influenzae type $\mathbf{B}$ conjugate vaccines appear to be equally very effective in the U.S. when given at 18 months of age and probably at 15 months as well. Studies to document that efficacy are currently in progress.

Because $H$. influenzae infections occur in children as young as 6 to 8 weeks, a major goal is to develop vaccines that will be effective at this early age. Toward that goal, the FDA is deliberating over the immunogenicity data of the conjugate vaccines and considering licensure for younger children. In addition, studies are in progress evaluating multiple doses of conjugate vaccine, beginning at early ages. In a recent study from Finland, after three doses of PRP-D by 6 months, efficacy was $89 \%$. Disappointingly, however, in a recent study from Alaska, also after three doses of PRP-D by 6 months, the efficacy was only $37 \%$. Studies in progress are evaluating PRP-CRM and PRP-

NOMP at these early ages and the results should be available by the end of 1990. Immunogenicity results suggest that these two vaccines may be 
May 1990

more effective at earlier ages than PRP-D.

\section{Bibliography \\ American Academy of Pediatrics, Com- mittee on Infectious Diseases: Haemo- philus influenzae type $\mathrm{b}$ conjugate vaccines: Update. Pediatr 84:386-387, 1989. \\ Daum RS, Sood SK, Osterholm MT, et al: Decline in serum antibody to the capsule of Haemophilus influenzae type $\mathrm{b}$ in the immediate post-immunization period. J Pediatr 114:742-747, 1989.}

Eskola J, Peltola H, Takala AK, et al: Efficacy of Haemophilus influenzae type b polysaccharide-diphtheria toxoid conjugate vaccine in infancy. $\mathbf{N}$ Engl $\mathbf{J}$ Med 317:717-722, 1987.

Marchant CD, Band E, Froeschie JE, et al: Depression of anticapsular antibody after immunization with Haemophilus influenzae type $b$ polysaccharide-diphtheria conjugate vaccine. Pediatr Infect Dis J 8:508-511, 1989.

Shapiro ED, Capobianco LA, Berg AT, et al: The immunogenicity of Haemophilus influenzae type $\mathrm{b}$ polysac-
charide-Neisseria meningitidis group B outer membrane protein complex vaccine in infants and young children. $J$ Infect Dis 160:1064, 1989.

Ward JI, Brenneman G, Lepow M: Haemophilus influenzae type $b$ anticapsular antibody responses to PRP-pertussis and PRP-D vaccines in Alaska native infants. J Infect Dis 158:719-723, 1988.

Weinberg GA, Granoff DM: Polysaccharide-protein conjugate vaccines for the prevention of Haemophilus influenzae type $\mathrm{b}$ disease. J Pediatr 113:621-631, 1988.

\section{CASE REPORT}

A 16-year-old white female presented to her local physician 1 week after stepping on a nail through her tennis shoe. The patient sought no medical care immediately following the injury, but reportedly cleaned the wound. On presentation, she had a low-grade fever $\left(101.2^{\circ} \mathrm{F}\right)$, a limp, local tenderness over the puncture wound site, and complaints of throbbing foot pain. The foot was swollen and red over the plantar surface with a $2 \times 3$ $\mathrm{cm}$ area of cellulitis. The local physician began ceftriaxone, $2 \mathrm{~g}$ twice a day, for foot cellulitis and followed her as an outpatient. On day 4 of therapy, the foot had become diffusely swollen, cool to touch, markedly tender, and the area of cellulitis had grown to $4 \times 5 \mathrm{~cm}$. She had also begun to experience right upper quadrant pain, nausea, vomiting, and anorexia on the morning of day 4 of ceftriaxone therapy. She was referred for an unresponsive foot cellulitis.

On referral, the examination of the foot revealed an area of cellulitis with fluctuance and marked tenderness over the second metatarsophalangeal joint with movement. The admitting physician made the tentative diagnosis of Pseudomonas osteochondritis/ septic arthritis and a subcutaneous abscess/cellulitis probably due to a staphylococcal wound infection. The ceftriaxone was discontinued and she was treated with intravenous nafcillin,
$1 \mathrm{~g}$ every 6 hours, and tobramycin, 60 mg every 8 hours.

Orthopedic surgery was consulted and she was taken to surgery on the same day. A subcutaneous abscess containing 4 to $6 \mathrm{~mL}$ of purulent drainage grew Pseudomonas aeruginosa and Staphylococcus aureus. The second metatarsal head was involved and the metatarsophalangeal joint was infected. Cultures of the cartilage/bone scrapings and joint fluid grew $P$. aeruginosa. Both Pseudomonas isolates were sensitive to tobramycin and resistant to ceftriaxone.

Further investigation of the abdominal complaints revealed a tender right upper quadrant and positive Murphy's sign. Her liver function studies were elevated ( $\gamma \mathrm{GT}$, ten times normal; SGOT, two times normal). An ultrasound of her liver and gallbladder revealed hyperechogenic sludge in the gallbladder.

She promptly defervesced postoperatively with resolution of her pain and foot complaints and good healing; she was discharged from the hospital after 7 days of intravenous antibiotics on an additional 3 days of oral dicloxacillin for the resolving cellulitis. Seven days after discontinuation of ceftriaxone, she had resolved her anorexia, nausea, vomiting, and abdominal pain. Her liver function tests and gallbladder ultrasound had re- turned to normal by 8 weeks postdischarge.

\section{Discussion}

This case report illustrates two important areas recently described in the literature. The issue of Pseudomonas osteochondritis following a nail puncture wound to the foot has recently been updated by a prospective, 10 year evaluation of 77 patients. In this series, the emphasis of early recognition from historical data and diagnosis by aggressive sampling for cultures was highlighted by the demonstration that Pseudomonas osteochondritis is a surgical disease. Following appropriate drainage of subcutaneous abscesses, curettage of infected bone/cartilage, and drainage plus irrigation of infected joints, these patients were treated with intravenous anti-Pseudomonas antibiotics for 7.5 \pm 1.2 days with a successful outcome. Only two relapses (2/77) were found in $5.2 \pm 3.4$ years follow-up with both relapses due to previously undiagnosed septic arthritis. These children were discharged requiring no antibiotic therapy for Pseudomonas. This is fortunate because no effective oral therapy with tissue penetration exists for children with the current restrictions on the use of oral fluoroquinolones. This prospective study follows the author's previous retro- 